\title{
ERRATUM
}

\section{Streptococcus pyogenes and phagocytic killing}

U. von Pawel-Rammingen, B.P. Johansson, H. Tapper \& L. Björck

Nature Med. 8, 1043-1046 (2002).

Page 1044, Fig. $1 b$ is incorrect. The correct panels are shown below.
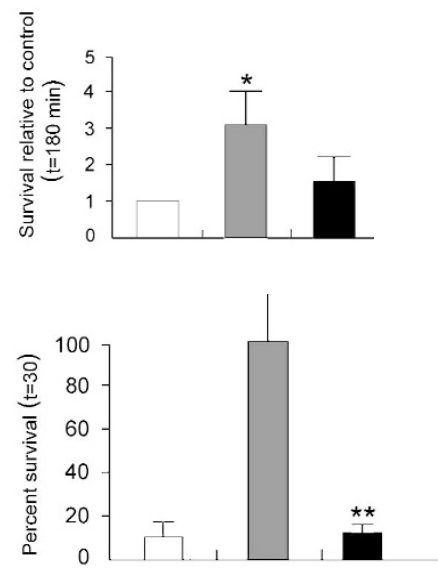

We regret this error.

\section{CORRIGENIDA}

Loss of HIF-2 $\alpha$ and inhibition of VEGF impair fetal lung maturation, whereas treatment with VEGF prevents fatal respiratory distress in premature mice

V. Compernolle, K. Brusselmans, T. Acker, P. Hoet, M. Tjwa, H. Beck, S. Plaisance, Y. Dor, E. Keshet, F. Lupu, B. Nemery, M. Dewerchin, P. Van Veldhoven, K. Plate, L. Moons, D. Collen \& P. Carmeliet

Nature Med. 8, 702-710 (2002). Published online : 10 June 2002; doi:10.1038/nm721

Page 704, credit for the schematic panels in the model of vessel remodeling during lung maturation (Fig. 2c-e) was omitted. The panels were originally published in Fig. 3 of Burri, P.H. Fetal and postnatal development of the lung. Annu. Rev. Physiol. 46, 617-628 (1984). This reference should be added as reference 44 on p. 710 .

Elsewhere on the same page, the authors erroneously used the term 'alveoli', which refers to structures that form only after birth, to denote 'saccules', which form during the saccular stage of embryonic development. In the legend of Fig. 2, the description of parts $c-e$ should read: " $c-\boldsymbol{e}$, Schematic illustration of capillary remodeling in septa. During lung development, capillaries around the airspaces establish a close contact with the overlying cuboidal epithelium $(c)$. Perinatally, secondary septa develop from primary septa, containing a double capillary network $(d)$. In the mature lung, interalveolar septa contain a single capillary layer $(e)$." In the body of the article, the second through fourth full sentences of the right-hand column should be replaced by the following: "In addition, capillaries in the septa failed to remodel properly in HIF- $2 \alpha^{-1-}$ mice. During normal lung development ${ }^{44}$, capillaries, which previously formed a loose network within the mesenchyme, arrange themselves around the airspaces, subsequently establishing in many places a close contact with the overlying cuboidal epithelium (Fig. 2c). During the saccular stage, the capillary networks form a capillary bilayer in the intersacullar septa as the airspaces approach each other (Fig. $2 d$ ). In the mature lung, interalveolar septa contain a single capillary layer (Fig. $2 e$ )."

Sphingosine 1-phosphate preserves fertility in irradiated female mice without propagating genomic damage in offspring F. Paris, G.I. Perez, Z. Fuks, A. Haimovitz-Friedman, H. Nguyen, M. Bose, A. Ilagan, P.A. Hunt, W.F. Morgan, J.L. Tilly \& R. Kolesnick

Nature Med. 8, 901-903 (2002).

Page 902, information was omitted from the affiliation listing. A footnote should have been included referring to authors F. Paris and G.I. Perez stating that these two authors contributed equally to this work.

The authors regret these errors. 


\section{ERRATUM}

Streptococcus pyogenes and phagocytic killing

U. von Pawel-Rammingen, B.P. Johansson, H. Tapper \& L. Björck

Nature Med. 8, 1329 (2002).

The corrected panels originally published in this erratum were incorrect.

The correct panels appear below.
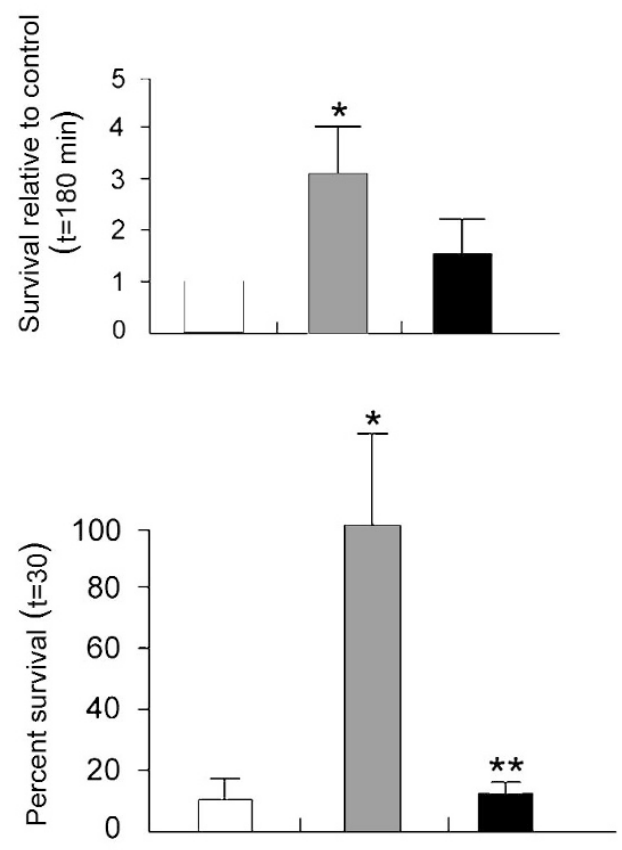

We regret this error. 